Pacific Journal of Mathematic 


\section{THE DETERMINANTAL IDEALS OF LINK MODULES. II}

\section{LORENZO TRALDI}

Let $H$ be the multiplicative free abelian group of rank $m \geq 1$. Suppose $0 \rightarrow B \rightarrow A \rightarrow I H \rightarrow 0$ is a short exact sequence of $\mathrm{Z} H$-modules, and the module $A$ is finitely generated. Then $B$ is also a finitely generated $\mathbf{Z} H$-module, and for any $k \in \mathbf{Z}$ the determinantal ideals of $A$ and $B$ satisfy the equality

$$
E_{k}(A):(I H)^{p}=E_{k-1}(B):(I H)^{q}
$$

for all sufficiently large values of $p$ and $q$. Furthermore, if this exact sequence is the link module sequence of a tame link of $m$ components in $S^{3}$, then

$$
E_{k}(A)=E_{k-1}(B):(I H)^{\left(\frac{m-1}{2}\right)}
$$

whenever $k \geq m$.

1. Introduction. Let $H$ be the multiplicative free abelian group of rank $m \geq 1$, and $\mathbf{Z} H$ its integral group ring; if $\varepsilon: \mathbf{Z} H \rightarrow \mathbf{Z}$ is the augmentation map then its kernel is the augmentation ideal $I H$ of $\mathbf{Z H}$. Following [6], we will call a short exact sequence

$$
0 \rightarrow B \stackrel{\phi}{\rightarrow} A \stackrel{\psi}{\rightarrow} I H \rightarrow 0
$$

of $\mathbf{Z} H$-modules and homomorphisms an augmentation sequence, provided that the $\mathbf{Z} H$-module $A$ is finitely generated. The module $B$ is then also finitely generated, and so for any $k \in \mathbf{Z}$ there are well-defined determinantal ideals $E_{k}(A), E_{k}(B) \subseteq \mathbf{Z} H$.

In [6] we discussed the relationship between the product ideals $E_{k}(A) \cdot(I H)^{p}$ and $E_{k-1}(B) \cdot(I H)^{q}$ for various values of $k, p$, and $q$. In the present paper, instead, we will consider the relationship between the various quotient ideals $E_{k}(A):(I H)^{p}$ and $E_{k-1}(B):(I H)^{q}$. (We recall the definition: if $U, V \subseteq \mathbf{Z H}$ are ideals then the quotient ideal $U: V$ is $\{x \in \mathbf{Z} H \mid x V \subseteq U\}$.)

At first glance, it may seem that if $U \subseteq \mathbf{Z H}$ is an ideal the quotient ideals $U:(I H)^{p}$ and the various product ideals $U \cdot(I H)^{q}$ are, in some 
sense, "duals" of each other, but this is not so. For the descending sequence

$$
U=U \cdot(I H)^{0} \supseteq U \cdot(I H)^{1} \supseteq U \cdot(I H)^{2} \supseteq \cdots
$$

of ideals of $\mathbf{Z H}$ need not terminate, in general, while since $\mathbf{Z H}$ is noetherian the ascending sequence

$$
U=U:(I H)^{0} \subseteq U:(I H)^{1} \subseteq U:(I H)^{2} \subseteq \cdots
$$

must, that is, there is a (unique least) $\rho(U)$ such that

$$
U:(I H)^{\rho(U)}=U:(I H)^{r} \quad \forall r \geq \rho(U) .
$$

We will devote most of our attention to this terminal quotient ideal.

THEOREM (1.1). If (1) is an augmentation sequence then for any $k \in \mathbf{Z}$

$$
E_{k}(A):(I H)^{\rho\left(E_{k}(A)\right)}=E_{k-1}(B):(I H)^{\rho\left(E_{k-1}(B)\right)} .
$$

It is of interest, then, to determine the integers $\rho\left(E_{k}(A)\right)$ and $\rho\left(E_{k-1}(B)\right)$. Though this seems impracticable in general, we will prove

TheOREM (1.2). If (1) is an augmentation sequence, $n \in \mathbf{Z}$, and $\varepsilon E_{n}(A)$ $=\mathbf{Z}$, then $\rho\left(E_{k}(A)\right)=0$ whenever $k \geq n$. Furthermore, $\rho\left(E_{k-1}(B)\right)=0$ whenever $k \geq n+\left(\begin{array}{c}m-1 \\ 2\end{array}\right)$, and $\rho\left(E_{k-1}(B)\right) \leq n+\left(\begin{array}{c}m-1 \\ 2\end{array}\right)-k$ whenever $n \leq$ $k \leq n+\left(\begin{array}{c}m-1 \\ 2\end{array}\right)$. Consequently, $\rho\left(E_{k-1}(B)\right) \leq\left(\begin{array}{c}m-1 \\ 2\end{array}\right)$ whenever $k \geq n$.

(Here $\left(\begin{array}{c}m-1 \\ 2\end{array}\right)$ is the binomial coefficient, and in particular $\left(\begin{array}{l}0 \\ 2\end{array}\right)=\left(\begin{array}{l}1 \\ 2\end{array}\right)=0$.)

If (1) is the module sequence of a tame link $L \subseteq S^{3}$ of $m$ components (described, e.g., in [1]) then it is known [5] that $\varepsilon E_{m}(A)=\mathbf{Z}$. (Note: in [5] the notation $E_{k}(A)=E_{k}(L)$ is used in this case.) Combining this with Theorems (1.1) and (1.2), we obtain then

COROLlary (1.3). If (1) is the module sequence of a tame link $L \subseteq S^{3}$,

$$
E_{k}(A)=E_{k-1}(B)
$$

whenever $k>\left(\begin{array}{c}m \\ 2\end{array}\right)$, and

$$
E_{k}(A)=E_{k-1}(B):(I H)^{m+\left(\begin{array}{c}
m-1 \\
2
\end{array}\right)-k}
$$

whenever $m \leq k \leq\left(\begin{array}{c}m \\ 2\end{array}\right)$. Consequently,

$$
E_{k}(A)=E_{k-1}(B):(I H)^{\left(\begin{array}{c}
m-1 \\
2
\end{array}\right)}
$$

whenever $k \geq m$. 
A special case of this is particularly pleasant: if (1) is the module sequence of a tame two-component link $L \subseteq S^{3}$ then $E_{k}(A)=E_{k-1}(B)$ whenever $k \geq 2$. Since $E_{1}(A)=E_{0}(B) \cdot I H$, and $E_{k}(A)=E_{k-1}(B)=0$ whenever $k \leq 0$, it follows that for any $k \in \mathbf{Z} E_{k}(A)$ and $E_{k-1}(B)$ are equivalent as invariants of $L$, that is, each ideal determines the other. In this respect, the behavior of these invariants for two-component links is analogous to their behavior for knots. (Recall that if $m=1$ and (1) is any augmentation sequence then [6] $E_{k}(A)=E_{k-1}(B)$ for every value of $k$.)

For links of three or more components in $S^{3}$, the relationship between the determinantal ideals of the modules $A$ and $B$ appearing in the link module sequence is more complex; we will discuss this further in $\S 3$.

Another result, analogous to Theorem (1.2) (though seemingly of less use in the application to the module sequences of tame links), is

THEOREM (1.4). If (1) is an augmentation sequence, $n \in \mathbf{Z}$, and $\varepsilon E_{n-1}(B)=\mathbf{Z}$, then $\rho\left(E_{k-1}(B)\right)=0$ whenever $k \geq n$. Furthermore, $\rho\left(E_{k}(A)\right)=0$ whenever $k \geq n+m-1$, and $\rho\left(E_{k}(A)\right) \leq n+m-1-k$ whenever $n \leq k \leq n+m-1$. Consequently, $\rho\left(E_{k}(A)\right) \leq m-1$ whenever $k \geq n$.

The author would like to express his gratitude to William S. Massey, for his stimulating correspondence, and to Sharon Richter, for her drawing.

\section{Proofs.}

Proposition (2.1). Let $U$ and $V$ be ideals of $\mathbf{Z} H$. Then $U:(I H)^{\rho(U)}=$ $V:(I H)^{\rho(V)}$ if, and only if, there are integers $p, q \geq 0$ such that $U \cdot(I H)^{p}$ $\subseteq V$ and $V \cdot(I H)^{q} \subseteq U$.

Proof. First, suppose that $U:(I H)^{\rho(U)}=V:(I H)^{\rho(V)}$. Then $U \cdot(I H)^{\rho(V)} \subseteq\left(U:(I H)^{\rho(U)}\right) \cdot(I H)^{\rho(V)}=\left(V:(I H)^{\rho(V)}\right) \cdot(I H)^{\rho(V)} \subseteq V$, and similarly $V \cdot(I H)^{\rho(U)} \subseteq U$.

Suppose, instead, that there are non-negative integers $p$ and $q$ as described. Then $\left(U:(I H)^{\rho(U)}\right) \cdot(I H)^{p+\rho(U)} \subseteq U \cdot(I H)^{p} \subseteq V$, and hence $U:(I H)^{\rho(U)} \subseteq V:(I H)^{p+\rho(U)} \subseteq V:(I H)^{\rho(V)}$. Similarly, $V:(I H)^{\rho(V)} \subseteq$ $U:(I H)^{\rho(U)}$, so these two ideals coincide.

Theorem (1.1) follows immediately from Proposition (2.1) and Theorem (1.1) of [6]. 
LeMMa (2.2). Let $U$ and $V$ be ideals of $\mathbf{Z} H$, and suppose that $\varepsilon(U)=\mathbf{Z}$. Then $U+V=U+V \cdot(I H)^{k}$ for any $k \geq 0$.

Proof. Since $(I H)^{0}=\mathbf{Z} H$, certainly $U+V=U+V \cdot(I H)^{0}$.

Since $\varepsilon(U)=\mathbf{Z}, \quad U+I H=\mathbf{Z} H$, and hence $U+V=(U+V)$. $(U+I H) \subseteq U+V \cdot I H \subseteq U+V$. Thus $U+V=U+V \cdot I H$.

Proceeding inductively, suppose $k \geq 1$ and $U+V=U+V \cdot(I H)^{k}$. Then $U+V=U+V \cdot(I H)^{k}=\left(U+V \cdot(I H)^{k}\right) \cdot(U+I H) \subseteq U+$ $V \cdot(I H)^{k+1} \subseteq U+V$, and hence $U+V=U+V \cdot(I H)^{k+1}$.

Corollary (2.3). Let $U \subseteq \mathbf{Z H}$ be an ideal with $\varepsilon(U)=\mathbf{Z}$. Then $\rho(U)=0$.

Proof. By definition, $\left(U:(I H)^{\rho(U)}\right) \cdot(I H)^{\rho(U)} \subseteq U$, and hence $U=U$ $+\left(U:(I H)^{\rho(U)}\right) \cdot(I H)^{\rho(U)}$. By the preceding lemma, then, $U=U+$ $\left(U:(I H)^{\rho(U)}\right)$, that is, $U \supseteq U:(I H)^{\rho(U)}$. Since $U \subseteq U:(I H)^{\rho(U)}$, it follows that $U=U:(I H)^{\rho(U)}$, and hence $\rho(U)=0$.

We may now proceed to the proof of Theorem (1.2); suppose (1) is an augmentation sequence and $\varepsilon E_{n}(A)=\mathbf{Z}$.

If $m=1$, then by Theorem $(1.1)_{1}$ of [6] $E_{k}(A)=E_{k-1}(B)$ for any value of $k$. Also, if $k \geq n$ then $E_{k}(A) \supseteq E_{n}(A)$, so $\varepsilon E_{k}(A)=\mathbf{Z}$, so by Corollary $(2.3) \rho\left(E_{k}(A)\right)=0$.

If $m=2$, then by Theorem $(1.1)_{2}$ of [6] $E_{k-1}(B) \cdot I H \subseteq E_{k}(A) \subseteq$ $E_{k-1}(B)$ for any value of $k \in \mathbf{Z}$. If $k \geq n$ then $E_{k}(A) \supseteq E_{n}(A)$, so by Corollary (2.3) $\rho\left(E_{k}(A)\right)=0$. Furthermore, since $E_{k-1}(B) \cdot I H \subseteq E_{k}(A)$, $E_{k}(A)=E_{k}(A)+E_{k-1}(B) \cdot I H$, so by Lemma (2.2) $E_{k}(A)=E_{k}(A)+$ $E_{k-1}(B)$, that is, $E_{k}(A) \supseteq E_{k-1}(B)$; since $E_{k}(A) \subseteq E_{k-1}(B)$, it follows that $E_{k}(A)=E_{k-1}(B)$.

If $m \geq 3$ and $k \geq n$ then $\mathbf{Z}=\varepsilon E_{n}(A)=\varepsilon E_{k}(A)$, so by Corollary (2.3) $\rho\left(E_{k}(A)\right)=0$. As shown in $\S 3$ of [6],

$$
E_{k-1}(B) \supseteq \sum_{i} E_{i+m}(X) E_{k-i-1}(A),
$$

where $X$ is a $\mathbf{Z} H$-module with $E_{m-2}(X)=0, E_{j}(X)=(I H)^{\left(\frac{m}{2}\right)-j}$ for $m-1 \leq j<\left(\begin{array}{c}m \\ 2\end{array}\right)$, and $E_{\left(\begin{array}{c}m \\ 2\end{array}\right)}(X)=\mathbf{Z} H$.

In particular, if $k \geq n+\left(\begin{array}{c}m-1 \\ 2\end{array}\right)$ then $E_{k-1}(B) \supseteq E_{\left(\begin{array}{c}m \\ 2\end{array}\right)}(X) E_{k-\left(\begin{array}{c}m-1 \\ 2\end{array}\right)}(A)$ $=E_{k-\left(\begin{array}{c}m-1 \\ 2\end{array}\right)}(A) \supseteq E_{n}(A)$, so $\varepsilon E_{k-1}(B)=\varepsilon E_{n}(A)=\mathbf{Z}$, so by Corollary $(2.3)$ $\rho\left(E_{k-1}(B)\right)=0$. 
If $n \leq k<n+\left(\begin{array}{c}m-1 \\ 2\end{array}\right)$, then

$$
\begin{aligned}
E_{k-1}(B) & \supseteq E_{k-n-1+m}(X) E_{n}(A)+E_{m-1}(X) E_{k}(A) \\
& =(I H)^{\left(\begin{array}{c}
m-1 \\
2
\end{array}\right)+n-k} \cdot E_{n}(A)+(I H)^{\left(\begin{array}{c}
m-1 \\
2
\end{array}\right)} \cdot E_{k}(A) \\
& =(I H)^{\left(\begin{array}{c}
m-1 \\
2
\end{array}\right)+n-k} \cdot\left(E_{n}(A)+(I H)^{k-n} \cdot E_{k}(A)\right) .
\end{aligned}
$$

Since $\varepsilon E_{n}(A)=\mathbf{Z}$, it follows from Lemma (2.2) that $E_{n}(A)+$ $(I H)^{k-n} \cdot E_{k}(A)=E_{n}(A)+E_{k}(A)$, so since $E_{n}(A) \subseteq E_{k}(A)$ (and hence $\left.E_{n}(A)=E_{n}(A)+E_{k}(A)\right)$ we conclude that

$$
E_{k-1}(B) \supseteq(I H)^{\left(\frac{m-1}{2}\right)+n-k} \cdot E_{k}(A) .
$$

Since $\rho\left(E_{k}(A)\right)=0$ (as noted earlier), it follows from this and Theorem (1.1) that

$$
E_{k-1}(B) \supseteq(I H)^{\left(\begin{array}{c}
m-1 \\
2
\end{array}\right)+n-k} \cdot\left(E_{k-1}(B):(I H)^{\rho\left(E_{k-1}(B)\right)}\right),
$$

and hence

$$
E_{k-1}(B):(I H)^{\rho\left(E_{k-1}(B)\right)} \subseteq E_{k-1}(B):(I H)^{\left(\begin{array}{c}
m-1 \\
2
\end{array}\right)+n-k} .
$$

That $\rho\left(E_{k-1}(B)\right) \leq\left(\begin{array}{c}m-1 \\ 2\end{array}\right)+n-k$ follows immediately.

This completes the proof of Theorem (1.2).

Turning to Theorem (1.4), suppose (1) is an augmentation sequence and $\varepsilon E_{n-1}(B)=\mathbf{Z}$.

If $m=1$, then by Theorem $(1.1)_{1}$ of [6] $E_{k}(A)=E_{k-1}(B)$ for any value of $k$. If $k \geq n$ then $E_{k-1}(B) \supseteq E_{n-1}(B)$, and so $\varepsilon E_{k-1}(B)=\mathbf{Z}$; by Corollary (2.3), then, $\rho\left(E_{k-1}(B)\right)=0$.

If $m \geq 2$ and $k \geq n$ then $\mathbf{Z}=\varepsilon E_{n-1}(B)=\varepsilon E_{k-1}(B)$, so by Corollary (2.3) $\rho\left(E_{k-1}(B)\right)=0$. Also, by Lemma (2.1) of [6]

$$
E_{k}(A) \supseteq \sum_{i} E_{k-l}(B) E_{i}(I H) .
$$

In [2] it is shown that $E_{0}(I H)=E_{0}\left(N_{2}(m)\right)=0, E_{j}(I H)=E_{j}\left(N_{2}(m)\right)=$ $(I H)^{m-j}$ for $1 \leq j<m$, and $E_{m}(I H)=E_{m}\left(N_{2}(m)\right)=\mathbf{Z} H .\left(N_{2}(m)\right.$ is a presentation matrix for $I H$, studied in [2].)

In particular, if $k \geq n+m-1$ then $E_{k}(A) \supseteq E_{k-m}(B) E_{m}(I H)=$ $E_{k-m}(B) \supseteq E_{n-1}(B)$, so $\varepsilon E_{k}(A)=\mathbf{Z}$, and hence by Corollary (2.3) $\rho\left(E_{k}(A)\right)=0$. 
If $n \leq k<n+m-1$, then

$$
\begin{aligned}
E_{k}(A) & \supseteq E_{n-1}(B) E_{k-n+1}(I H)+E_{k-1}(B) E_{1}(I H) \\
& =(I H)^{m-k+n-1} \cdot E_{n-1}(B)+(I H)^{m-1} \cdot E_{k-1}(B) \\
& =(I H)^{m-k+n-1} \cdot\left(E_{n-1}(B)+(I H)^{k-n} \cdot E_{k-1}(B)\right) .
\end{aligned}
$$

Since $\varepsilon E_{n-1}(B)=\mathbf{Z}$, it follows from Lemma (2.2) that

$$
E_{n-1}(B)+(I H)^{k-n} \cdot E_{k-1}(B)=E_{n-1}(B)+E_{k-1}(B)=E_{k-1}(B) ;
$$

hence

$$
E_{k}(A) \supseteq(I H)^{n+m-1-k} \cdot E_{k-1}(B) .
$$

Since $\rho\left(E_{k-1}(B)\right)=0$, it follows from this and Theorem (1.1) that

$$
E_{k}(A) \supseteq(I H)^{n+m-1-k} \cdot\left(E_{k}(A):(I H)^{\rho\left(E_{k}(A)\right)}\right) .
$$

We may conclude from this that $\rho\left(E_{k}(A)\right) \leq n+m-1-k$.

This completes the proof of Theorem (1.4).

We may note here, without going into detail, that Theorems (1.1), (1.2), and (1.4) hold in a broader context, with $\mathbf{Z} H$ replaced by an arbitrary noetherian commutative ring with unity $R$, and $I H$ replaced by the ideal of $R$ generated by the elements of some $R$-sequence $\left\{r_{1}, \ldots, r_{m}\right\}$. (The hypotheses $\varepsilon E_{n}(A)=\mathbf{Z}$ and $\varepsilon E_{n-1}(B)=\mathbf{Z}$ of Theorems (1.2) and (1.4) should be replaced by the equivalent hypotheses $\mathbf{Z} H=E_{n}(A)+I H$ and $\mathbf{Z} H=E_{n-1}(B)+I H$, respectively, prior to any such generalization.) An analogous generalization is discussed, in greater depth, in $\$ 5$ of [6].

3. Links of three or more components. A simple consequence of Corollary (1.3) is: if (1) is the module sequence of a tame link of $m$ components in $S^{3}$, then for $k \geq m$ the ideal $E_{k}(A)$ is determined by $E_{k-1}(B)$. A natural question to ask, especially in view of the cases $m=1$ and $m=2$ (discussed in $\S 1$ ) is: does $E_{k}(A)$, in turn, determine $E_{k-1}(B)$, for $k \geq m$ ? That the answer to this question is "no" may be seen by considering the three-component links $6_{2}^{3}$ and $8_{5}^{3}$ (as they are named in Appendix C of [4]). As W. S. Massey has shown, if (1) is the link module sequence of the former then $E_{3}(A)=\mathbf{Z} H$ and $E_{2}(B)=I H$ [3, Example 1], while if (1) is the link module sequence of the latter then $E_{3}(A)=\mathbf{Z H}$ $=E_{2}(B)[3$, Example 2].

Another natural question is: can the result of Theorem (1.1) be made more definitive for $1<k<m$, as it can for $k \geq m$ (Corollary (1.3)) and 
$k=1[2]$ ? Though we shall not answer this question, we will consider an example of a three-component link for which the relationship between $E_{2}(A)$ and $E_{1}(B)$ is particularly complex.

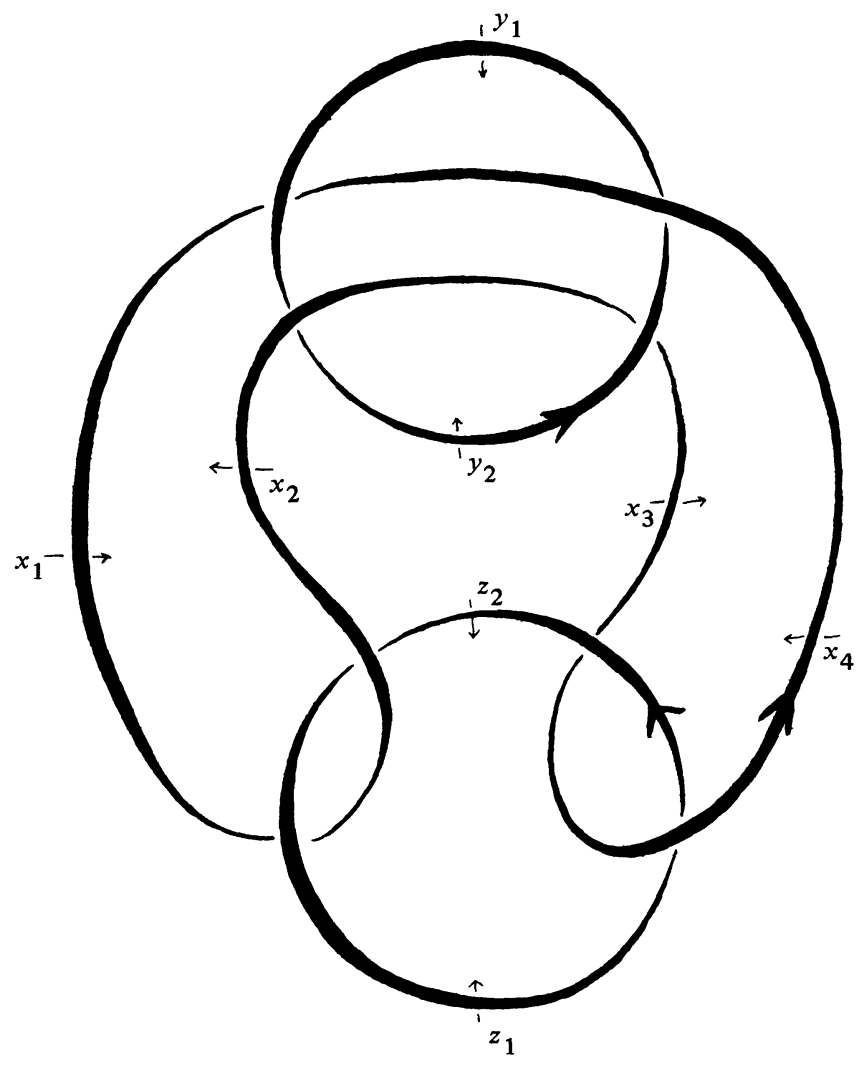

FIGURE 1

Pictured in Figure 1 is the link $8_{10}^{3}$ [4, Appendix C]. The Wirtinger presentation $[4$, p. 56] of the fundamental group $G$ of the complement of this link in $S^{3}$ is

$$
\begin{gathered}
\left\langle x_{1}, x_{2}, x_{3}, x_{4}, y_{1}, y_{2}, z_{1}, z_{2} ; x_{1} z_{1}=z_{1} x_{2}, y_{2} x_{2}=x_{3} y_{2},\right. \\
x_{3} z_{2}=z_{2} x_{4}, y_{1} x_{4}=x_{1} y_{1}, x_{2} y_{1}=y_{2} x_{2}, \\
\left.x_{4} y_{2}=y_{1} x_{4}, z_{1} x_{4}=x_{4} z_{2}, z_{2} x_{2}=x_{2} z_{1}\right\rangle .
\end{gathered}
$$

Since any one of the relations in this presentation is redundant, we may simply delete the seventh. Also, we may remove the fourth relation and the generator $x_{1}$, replacing any occurrence of $x_{1}$ in another relation by an occurrence of $y_{1} x_{4} y_{1}^{-1}$; similarly, we may remove the third relation and 
the generator $x_{3}$, replacing $x_{3}$ by $z_{2} x_{4} z_{2}^{-1}$ in the remaining relations. What results, after some simple rewriting of relations, is the presentation

$$
\begin{aligned}
\left\langle x_{2}, x_{4}, y_{1}, y_{2}, z_{1}, z_{2} ; x_{4}\right. & =y_{1}^{-1} z_{1} x_{2} z_{1}^{-1} y_{1}, y_{1}=x_{2}^{-1} y_{2} x_{2}, \\
x_{2}^{-1} y_{2}^{-1} z_{2} x_{4} z_{2}^{-1} y_{2} & \left.=1, x_{4} y_{2} x_{4}^{-1} y_{1}^{-1}=1, z_{1}=x_{2}^{-1} z_{2} x_{2}\right\rangle .
\end{aligned}
$$

After deleting the first relation and the generator $x_{4}$, and replacing $x_{4}$ by $y_{1}^{-1} z_{1} x_{2} z_{1}^{-1} y_{1}$ in the remaining relations, we may delete the second and fifth relations and the generators $y_{1}$ and $z_{1}$, substituting $x_{2}^{-1} y_{2} x_{2}$ for $y_{1}$ and $x_{2}^{-1} z_{2} x_{2}$ for $z_{1}$, and obtain the presentation

$$
\begin{aligned}
\left\langle x_{2}, y_{2}, z_{2} ; x_{2}^{-1} y_{2}^{-1} z_{2} x_{2}^{-1} y_{2}^{-1} z_{2} x_{2} z_{2}^{-1} y_{2} x_{2} z_{2}^{-1} y_{2}=1,\right. \\
\left.y_{2}^{-1} z_{2} x_{2} z_{2}^{-1} y_{2} x_{2} y_{2} x_{2}^{-1} y_{2}^{-1} z_{2} x_{2}^{-1} z_{2}^{-1}=1\right\rangle .
\end{aligned}
$$

The Alexander matrix $M$ of this presentation $[1, \S 3]$ is the transpose of the matrix

$$
\left(\begin{array}{cc}
\left(1+t_{1}^{-1} t_{2}^{-1} t_{3}\right)\left(t_{1}^{-1} t_{2}^{-1} t_{3}-t_{1}^{-1}\right) & \left(1-t_{2}\right)\left(t_{1}+t_{2}^{-1} t_{3}\right) \\
\left(1-t_{1}^{-1}\right)\left(t_{2}^{-1}+t_{1}^{-1} t_{2}^{-2} t_{3}\right) & \left(t_{1}-1\right)\left(t_{1}+t_{2}^{-1}\right) \\
\left(t_{1}^{-1}-1\right)\left(t_{2}^{-1}+t_{1}^{-1} t_{2}^{-2} t_{3}\right) & \left(t_{1}-1\right)\left(1-t_{2}^{-1}\right)
\end{array}\right) .
$$

(Here $t_{1}, t_{2}$, and $t_{3}$ are the elements of $G / G^{\prime}=H$ determined by the elements of $G$ represented by $x_{2}, y_{2}$, and $z_{2}$, respectively.) If (1) is the module sequence of the link $8_{10}^{3}$, then $M$ is a presentation matrix for the $\mathbf{Z} H$-module $A$ [1, §3], and hence, in particular, the ideal of $\mathbf{Z} H$ generated by the entries of $M$ is

$$
E_{2}(A)=\left(1+t_{1}^{-1} t_{2}^{-1} t_{3}\right) \cdot I H+\left(t_{1}+1, t_{2}-1\right) \cdot\left(t_{1}-1\right) .
$$

The matrix $M$ can be factored as a product $M=M^{\prime} \cdot N_{2}(3)$, where

$$
N_{2}(3)=\left(\begin{array}{ccc}
1-t_{2} & t_{1}-1 & 0 \\
1-t_{3} & 0 & t_{1}-1 \\
0 & 1-t_{3} & t_{2}-1
\end{array}\right)
$$

and

$$
M^{\prime}=\left(\begin{array}{ccc}
t_{1}^{-1} t_{2}^{-1}\left(1+t_{1}^{-1} t_{2}^{-1} t_{3}\right) & -t_{1}^{-1} t_{2}^{-1}\left(1+t_{1}^{-1} t_{2}^{-1} t_{3}\right) & 0 \\
t_{1}+t_{2}^{-1} t_{3} & 0 & t_{2}^{-1}\left(t_{1}-1\right)
\end{array}\right) .
$$


(Here $N_{2}(3)$ is a matrix discussed by Crowell and Strauss [2], with columns corresponding to the integers 1,2 , and 3 (in order), and rows corresponding to the pairs 12,13 , and 23 (in order).) It follows [2, p. 106] that the module $B$ of the link module sequence of $8_{10}^{3}$ has the presentation matrix

$$
\begin{aligned}
P & =\left(\begin{array}{c}
M^{\prime} \\
N_{3}(3)
\end{array}\right) \\
& =\left(\begin{array}{ccc}
t_{1}^{-1} t_{2}^{-1}\left(1+t_{1}^{-1} t_{2}^{-1} t_{3}\right) & -t_{1}^{-1} t_{2}^{-1}\left(1+t_{1}^{-1} t_{2}^{-1} t_{3}\right) & 0 \\
t_{1}+t_{2}^{-1} t_{3} & 0 & t_{2}^{-1}\left(t_{1}-1\right) \\
t_{3}-1 & 1-t_{2} & t_{1}-1
\end{array}\right) .
\end{aligned}
$$

$\left(N_{3}(3)\right.$ is another matrix discussed in [2]; its columns correspond to the pairs 12, 13, and 23 (in order).) Thus the ideal of $\mathrm{ZH}$ generated by the determinants of the two-by-two submatrices of $P$ is

$$
E_{1}(B)=E_{2}(A)+\left(1+t_{1}^{-1} t_{2}^{-1} t_{3}\right)^{2} .
$$

In particular, the $\mathbf{Z} H$-modules $A$ and $B$ of the link module sequence of $8_{10}^{3}$ have the property that

$$
\begin{aligned}
\left(E_{2}(A): I H\right) \cdot I H & =\left(E_{1}(B): I H\right) \cdot I H \\
& \subset E_{2}(A) \subset E_{1}(B) \subset E_{2}(A): I H=E_{1}(B): I H,
\end{aligned}
$$

in which all three indicated inclusions are strict. The relationship between $E_{2}(A)$ and $E_{1}(B)$ does not, then, seem to fall into the pattern of the simple relationships between $E_{k}(A)$ and $E_{k-1}(B)$ for $k \neq 2$ (namely, $E_{k}(A)=E_{k-1}(B) \cdot I H$ for $k<2$, and $E_{k}(A)=E_{k-1}(B): I H$ for $k>2$ ).

\section{REFERENCES}

1. R. H. Crowell, The derived module of a homomorphism, Advances in Math., 6 (1971), 210-238.

2. R. H. Crowell and D. Strauss, On the elementary ideals of link modules, Trans. Amer. Math. Soc., 142 (1969), 93-109.

3. W. S. Massey, Completion of link modules, Duke Math. J., 47 (1980), 399-420.

4. D. Rolfsen, Knots and Links, Publish or Perish, Inc., Berkeley, California, 1976.

5. L. Traldi, A generalization of Torres' second relation, Trans. Amer. Math. Soc., 269 (1982), 593-610.

6. The determinantal ideals of link modules, I, Pacific J. Math., 101 (1982), 215-222.

Received January 27, 1982. 



\section{PACIFIC JOURNAL OF MATHEMATICS}

EDITORS

Donald BABBITt (Managing Editor)

University of California

Los Angeles, CA 90024

Hugo Rossi

University of Utah

Salt Lake City, UT 84112

C. C. Moore and Arthur Ogus

University of California

Berkeley, CA 94720

\section{J. DugundiI}

Department of Mathematics

University of Southern California

Los Angeles, CA 90089-1113

R. Finn and H. SAMELSON

Stanford University

Stanford, CA 94305

\section{ASSOCIATE EDITORS}
R. ARENS
E. F. BECKENBACH
B. H. NEUMANN
F. WOLF
K. YOSHIDA (1906-1982)

\section{SUPPORTING INSTITUTIONS}

UNIVERSITY OF ARIZONA

UNIVERSITY OF BRITISH COLUMBIA

CALIFORNIA INSTITUTE OF TECHNOLOGY

UNIVERSITY OF CALIFORNIA

MONTANA STATE UNIVERSITY

UNIVERSITY OF NEVADA. RENO

NEW MEXICO STATE UNIVERSITY

OREGON STATE UNIVERSITY
UNIVERSITY OF OREGION

UNIVERSITY OF SOUTHERN CALIFORNIA

STANFORD UNIVERSITY

UNIVERSITY OF HAWAII

UNIVERSITY OF TOKYO

UNIVERSITY OF UTAH

WASHINGTON STATE UNIVERSITY

UNIVERSITY OF WASHINGTON 


\section{Pacific Journal of Mathematics}

Vol. 109, No. $1 \quad$ May, 1983

Donald George Babbitt and V. S. Varadarajan, Formal reduction theory of meromorphic differential equations: a group theoretic view $\ldots \ldots \ldots \ldots 1$

Jo-Ann Deborah Cohen, Norms on $F(X) \ldots \ldots \ldots \ldots \ldots \ldots \ldots \ldots \ldots \ldots$

Robert Fitzgerald, Witt kernels of function field extensions $\ldots \ldots \ldots \ldots \ldots 89$

Hervé Jacquet and Joseph Andrew Shalika, The Whittaker models of induced representations .............................. 107

Masakiti Kinukawa, Some generalizations of contraction theorems for

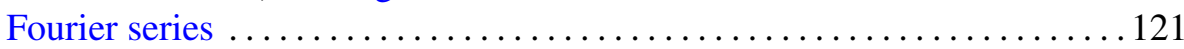

Joseph Weston Kitchen, Jr. and David A. Robbins, Sectional

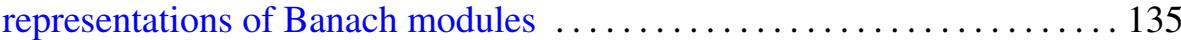

Victor Charles Pestien, Jr., Weak approximation of strategies in measurable gambling

Richard Scott Pierce and Charles Irvin Vinsonhaler, Realizing central

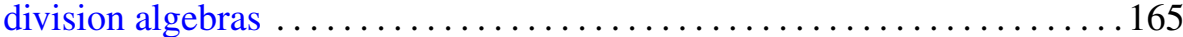

Walter Ricardo Ferrer Santos, Cohomology of comodules ............ 179

Marko Tadić, Harmonic analysis of spherical functions on reductive groups

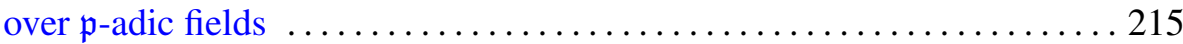

Lorenzo Traldi, The determinantal ideals of link modules. II . . . . . . . . 237

Alain J. Valette, A remark on the Kasparov groups $\operatorname{Ext}^{i}(A, B) \ldots \ldots \ldots 247$ 